\title{
Pick and Place Automation Machine Using PLC and Ladder Programming \\ ${ }^{*}$ Shubham Anjankar, ${ }^{2}$ Dr. Parag Jawarkar, ${ }^{3}$ Lokesh Heda, ${ }^{4}$ Vidya Dahake
${ }^{* 1,2,3}$ Department of Electronics Engineering. Shri Ramdeobaba College of Engineering and Management, Nagpur, INDIA
${ }^{4}$ Department of Electronics Engineering. J. D. College of Engineering and Management, Nagpur, INDIA \\ Email: "anjankarsc1@rknec.edu
}

Received: $20^{\text {th }}$ September 2018, Accepted: $11^{\text {th }}$ October 2018, Published: $31^{\text {st }}$ October 2018

\begin{abstract}
This paper aims at studying the development of a pick and place machine using PLC (programmable logic control). Originally, the tedious task of picking and placing the components of a product involved immense human labour. But nowadays industries are aiming at minimizing the human efforts by using automated optimized machines. These machines are all- in-one, multi-headed and multi-gantry. The pick and place automated machine when put into action directly places the product onto the next stage of production line. The software involves ladder logic programming and hardware involves pneumatic system, limit switches etc. Which would be further discussed in the paper. More productivity being the need of the hour, this machine considerably increases the speed of operation and significantly decreases human efforts.
\end{abstract}

\section{Keywords}

PLC, Ladder Logic , Vacuum Grippers, Sensor

\section{Introduction}

This paper discusses the working of Pick and Place Machine in the production line of a furniture industry. This machine is used to pick up panels using vacuum cup system from certain stacks and place it in a position where it is used for further processing [1].This was previously being done by human operators and to overcome the tedious and monotonous task of picking and placing the components of a product which involves immense human labour using automated optimized machines was a solution, which increases the production and reduces human effort.

Workers invest a huge amount of time in manually picking and placing products for the next stage of operation which can be utilized for other relevant operations. This propelled and motivated to design a mechanism that could eliminate these drawbacks [3, 4]. Presently, this machine is part of an industrially automated production line. Once this machine is put into action the machine will directly place panels onto the next stage of operation. This machine will considerably increase speed of operation. Thereby, making production more efficient leading to increasing profits to the industry [9]. PLC programming is used for the operation of this machine.

\section{Related Work}

Programmable Logic Control is a device that is on a greater scale used in the industry for interlocking of operations ,automation, ,event based control, and order of operations, is utilised in every process industry, requiring process safety and interlocks e.g. thermal power plant, steel industry, cement industry, pharmaceutical industry, petrochemical industry etc. A programmable logic controller, called a PLC is a computer- type device used to control the devices in an industrial facility. The PLCs control variety of industrial facilities E.g. Conveyor systems, food- processing machinery, auto assembly lines [1-6].

PLCs have gained preference on the factory floor and will remain on the top of the list of choices for some time to come. These devices were originally designed to replace relay logic circuits. The basic programming language, ladder diagram, resembles the same relay logic schematics. They are real-time controllers using cyclic behaviour. The ladder diagram and instruction list was the first programming language .Like mentioned earlier, the ladder diagram is historically connected with the relay logic used in control before programmable logic controllers emerged. The ladder diagram's basic structure looks like an electrical scheme, with contacts and coils connected between two power rails called rungs[7,8].

Pick and place automation machines have been built using IR1400 ROBOT, since 'Robots' the term is only a small subset of the technologies covered by the much broader term Automation'. Also this machine has been build with PLC 4DOF (degree of freedom). Pick and Place machine has become very important for the industrial based applications as part of the industrial line. 


\section{System Overview}

\section{Brief Discussion}

In this machine, there are 3 stacks from where the machine will pick-up the wooden planks and place the planks on a predecided home position where the planks will be used for the next stages of operation. The operator will have to give the input such as from which stack the planks have to be fetched and the number of planks that needs to be fetched and placed to the next stage. It consists of a beam on which a movable motor controlled arm is fixed. This arm has vacuum cups which pick up the panels [10-12].

\section{Broader Perspective}

The machine on power up finds its home position using certain predefined sequences. After this calibration, the system already has user input for the number of panels to be picked and which stack the panels should be picked from. The machine completes that operation. There are a few different modes of operation available in this machine where it lets the operator change certain parameters of operation. The machine is controlled by an AC servo motor, AC motor drive, PLC, limit switches and encoder.

\section{Algorithm}

- Power up and take input for stack number and number of panels

- Calibrate and Find Home Position

- Move towards entered stack

4 - Pickup panel from the stack and move towards home position then release

5 - Repeat above process for given input count

6 - Complete Cycles and then move to next stack as per priority

\section{7 - After completion, Return to home position}

\section{8 - Power down}

1. Initially, calibration for the machine takes place and the arm goes up and then moves rightmost direction , thus finding its home position(refer Fig 3.a.5 ).

2. After reaching the home position, it checks the input which specifies the stack number and the number of planks that needs to be transferred.

3. The arm then moves towards the stack and as it approaches the planks, it slows down due to the photoelectric sensor.

4. The vacuum cups hold the wooden planks and the arm then moves upwards and towards the stack to be placed in.

5. The arm then moves back to the home position for the process to continue till the given count ends.

6. Hence the job of placing the planks in the stacks is done with greater ease.

\section{Proposed Method}

The machine includes the hardware and the software part which will be discussed further.

\section{Hardware}

- This machine makes use of a pneumatic system, to hold the electronic arm at the top of the beam in a stable position.

- Pick and place machines uses vacuum grippers, which are simple astrictive devices, to hold large wooden planks provided the pretension surface is smooth enough to ensure suction.(refer fig F.a.1)

- The hardware consists of a speed limiting sensor which is used to decrease the speed when the machine arm reaches near the specified destination. The sensor is a photoelectric sensor. (Refer Fig 3.a.2)

- The machine arm travels towards to the destination with a specified speed and after detecting the sensor the speed decreases so that the machines doesn't crash and there is no damage to the machine 
- The limit switch also exists as the hardware part which is used to detect that the arm of the machine has reached exactly over the stack and now it has to fetch the planks. (Refer Fig 3.a.3)

- The motor in the design is a servomotor which drives the mechanical arm in linear motion i.e. in $\mathrm{x}$ and $\mathrm{z}$ plane (vertical and horizontal plane).

The interfacing of machine needs few electronic and electrical devices. The devices are (refer Fig 3.a.4):

- PLC: Programmable Logic Controller (PLC) is similar to a computer used in an industry to control manufacturing processes, such as in assembly lines, and robots. It is rugged, highly reliable, and easily programmable and fault diagnosis is comparatively easy.

- Transformer $(\mathrm{X})$ : A transformer is an electrical device that transfers electrical energy between two or more circuits through electromagnetic induction.

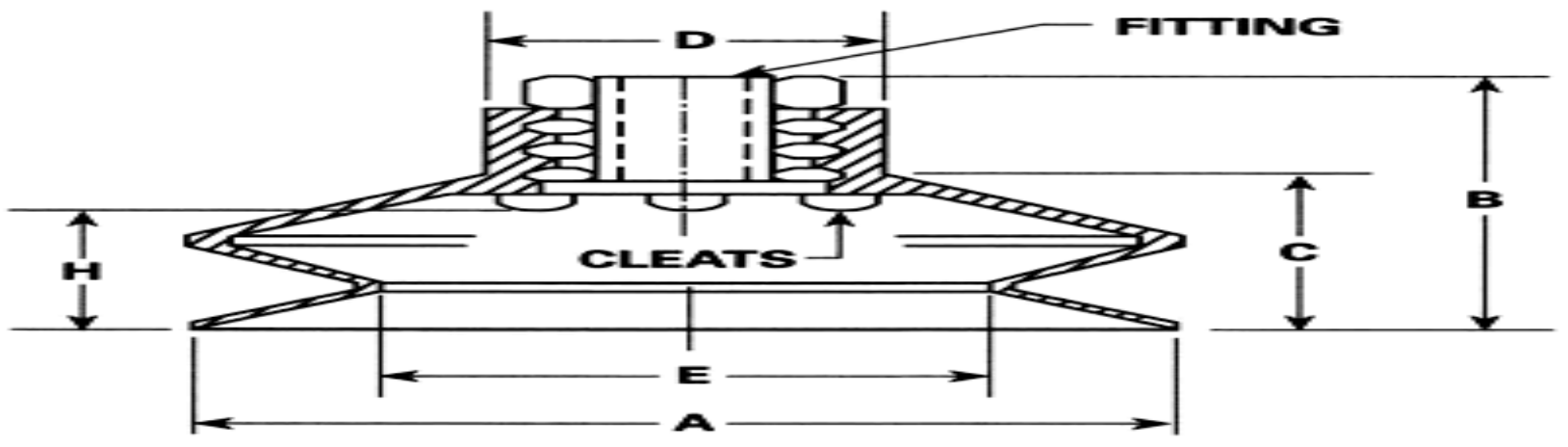

Figure 3.a.1: Vacuum Gripers

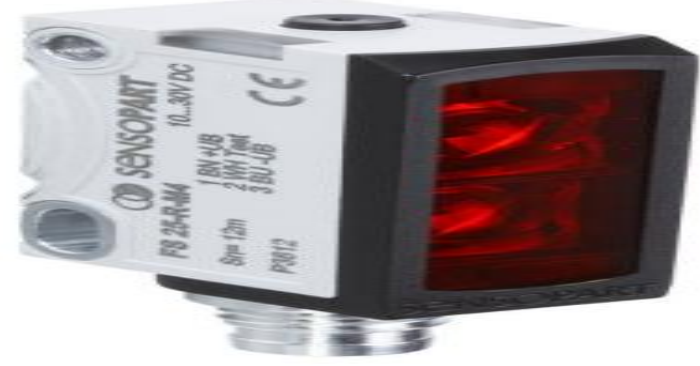

Figure 3.a.2: Photoelectric Sensor

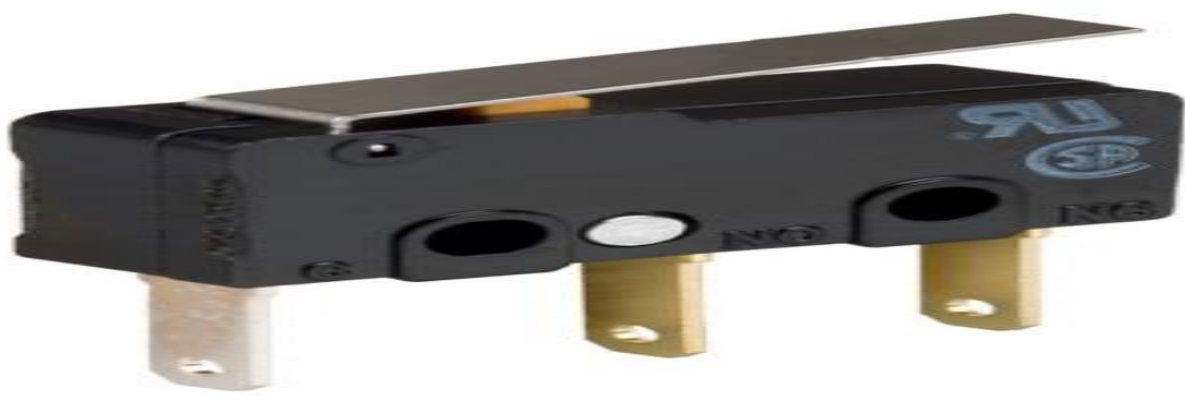

Figure 3.a.3: Limit Switches

- SMPS (PS-1): A switched-mode power supply is an electronic power supply that incorporates a switching regulator to convert electrical power efficiently. Here $230 \mathrm{~V}$ AC supply is converted into $24 \mathrm{~V}$ DC supply which is then given to PLC.

- SMPS Fan (F): The fan is used to cool the components in the SMPS. Since it sucks air out of the cabinet, it 
also helps reduce the temperature of the system.

- Relay Board (RB): Relay is an electrically operated switch. It is used to control a circuit by a separate lowpower signal and where multiple circuits are controlled by a single signal.

- Isolator: This switch is used to make sure that an electrical circuit is fully de-energized for service or device maintenance.

- MCB: Miniature circuit breaker is an automatically operated electrical switch designed to protect an electrical circuit from damage caused by excess current, typically resulting from an overload or short circuit.

- Servo drives: A servo drive is a special electronic amplifier used to power electric servomechanisms. A servo drive receives a command signal from a control system, amplifies the signal, and transmits electric current to a servo motor in order to produce motion proportional to the command signal.

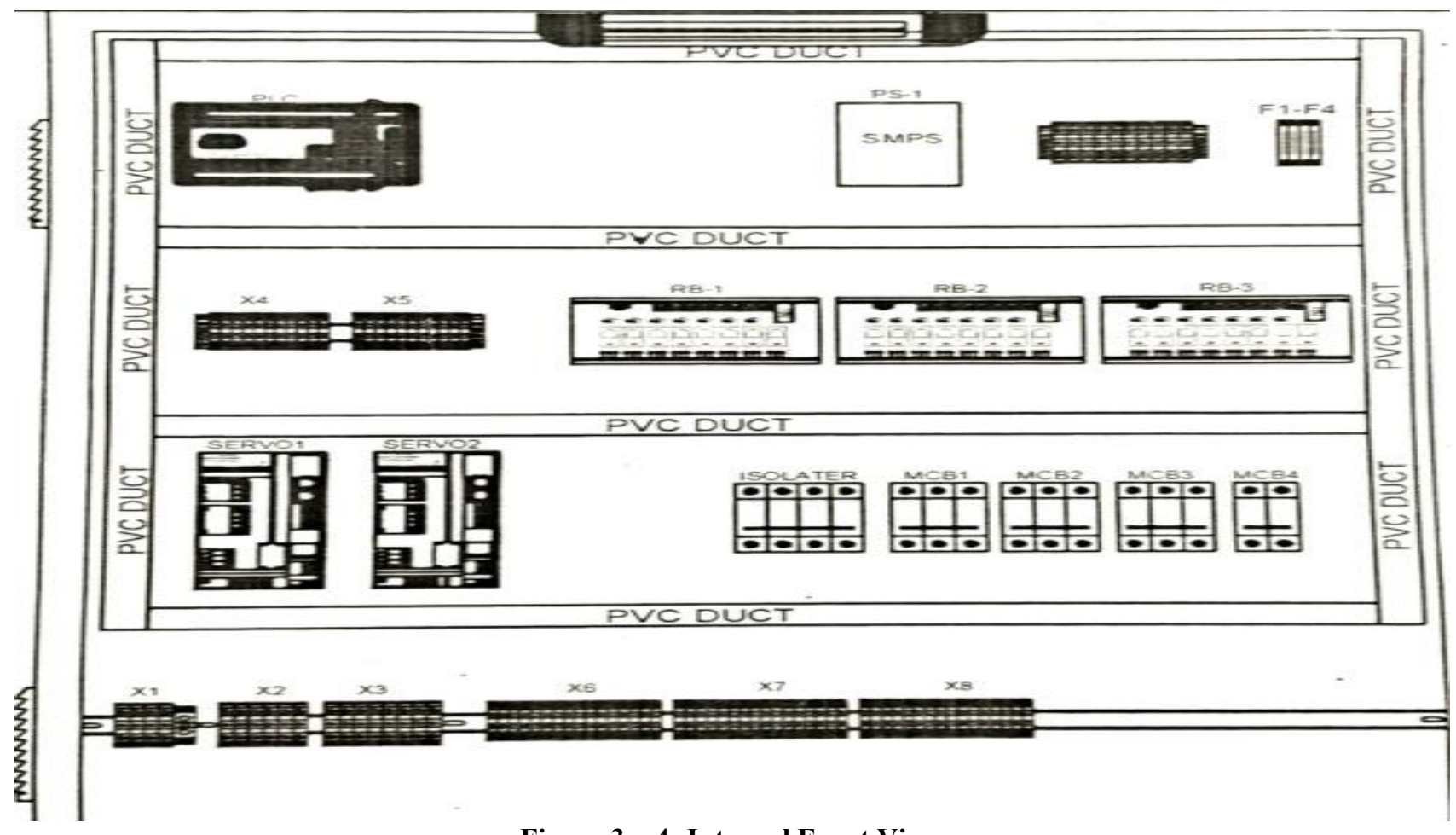

Figure 3.a.4: Internal Front View

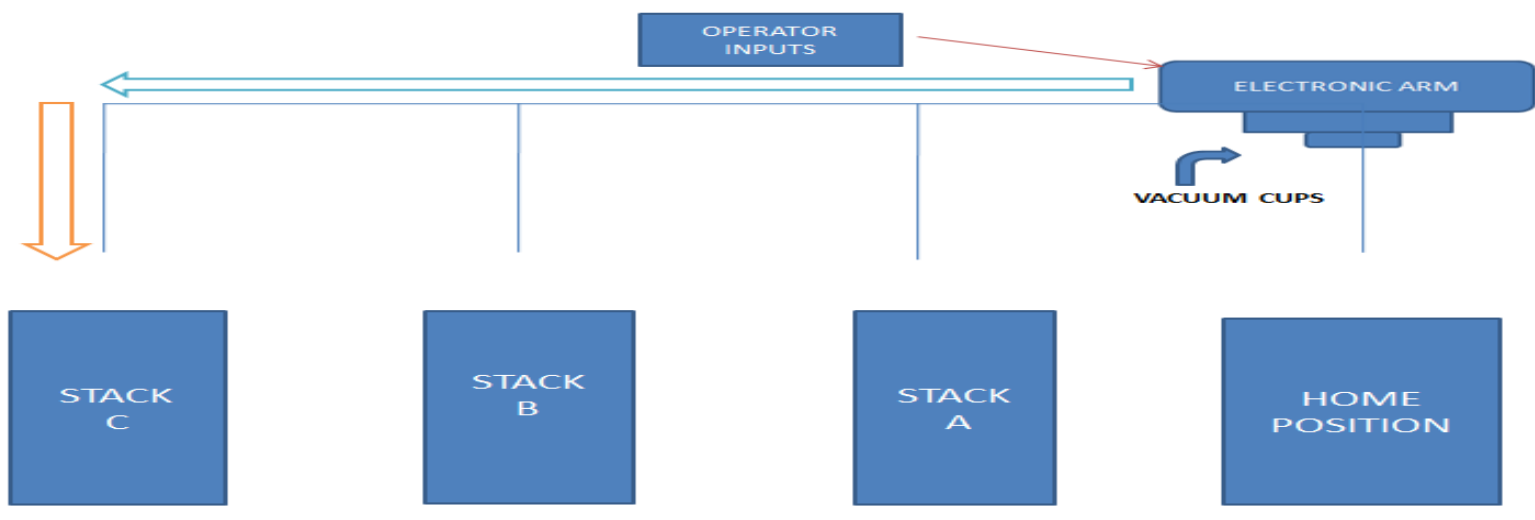

Figure 3.a.5: Schematic Representation of Machine 


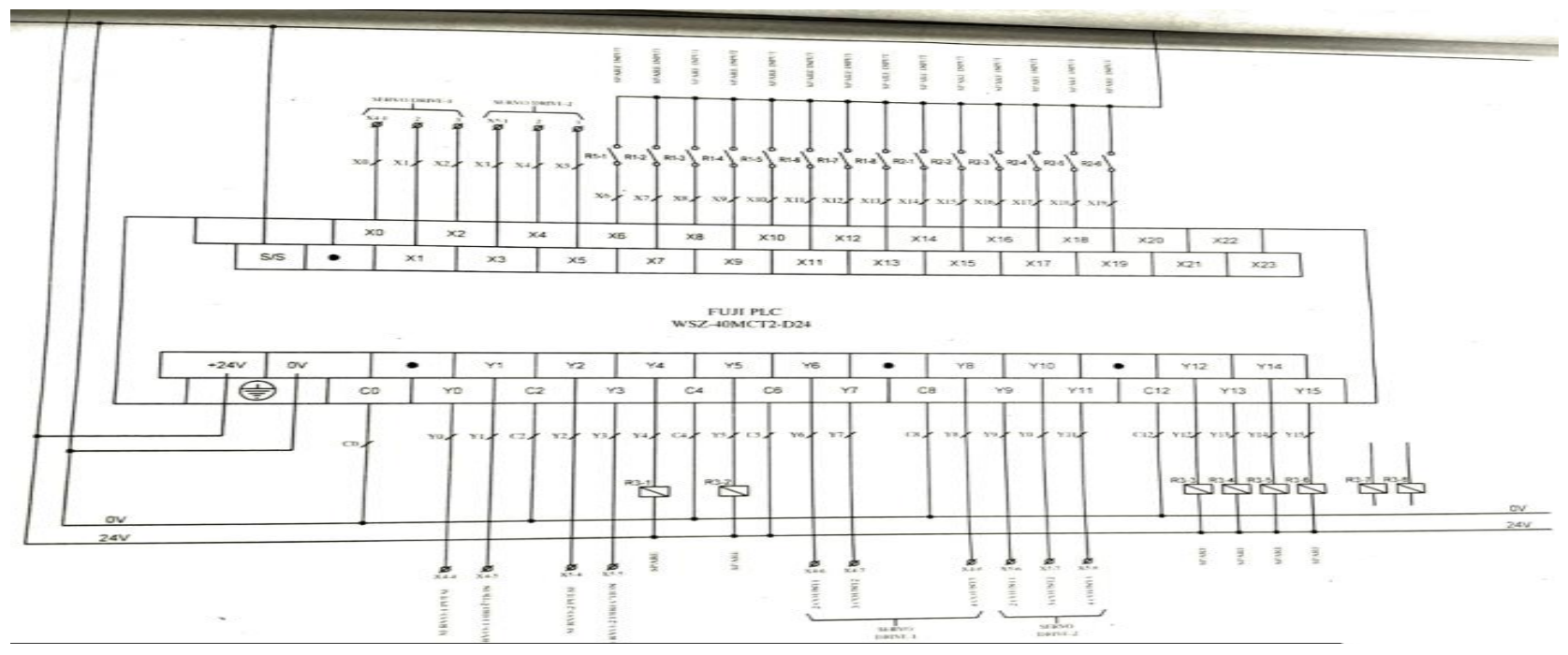

Figure 3.a.6: PLC Interfacing Circuit Diagram

\section{Block Diagram}

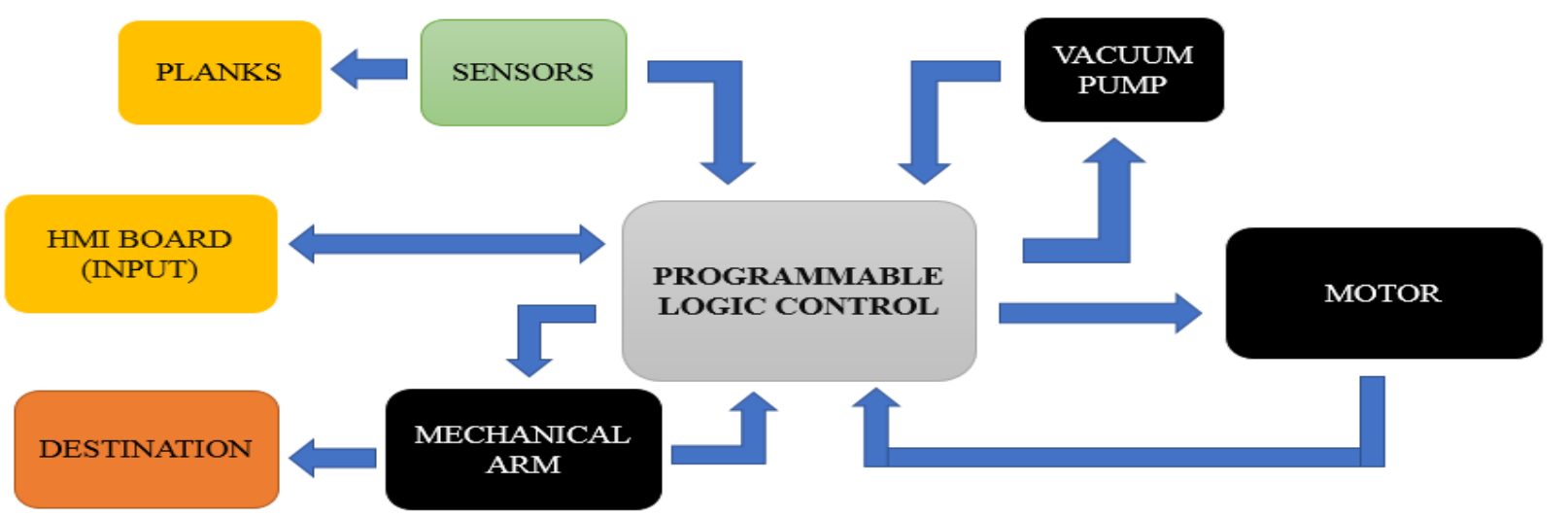

- The input is given to the PLC through the HMI Board, which states the stack number from where the planks are to be fetched and the number of planks to be fetched.

- After the input is detected the motor is switched on and mechanical arm starts moving towards the destination.

- Mechanical arm senses that it has reached the destination using encoder cycles and limit switches which acts as the sensors, this sensor output is given to the PLC, thus the PLC then instructs the motor to slow down, stop and turn on the vacuum to fetch the planks.

- The complete machine is a closed loop system.

Advantages of PLC over hardwired relay controller:

- Change in software design is much easier and cheaper as compared to change in hardware design.

- Less maintenance.

- The technicians and engineers can easily adapt to PLC because programming using ladder logic has no need of any kind of syntax, it is completely based on logic of the working.

- Advantages of PLC over microcontroller and microprocessor: Large capacity or Scalability, Modular Design Distributed architecture, Industrial Grade ,Interoperability of devices, Ladder Programming ,Safety, Utility, Easy Maintenance and repair, Inbuilt Functions and Algorithms ,Diagnostic Features

- The system is user programmable. It uses a language called "Relay Ladder" or RLL (Relay Ladder Logic). The name of this language implies that the control logic of the earlier days, which was built from relays, is being simulated. 


\section{Ladder Logic}

- Ladder Logic was a written method used originally to document the construction and design of relay racks used in process and manufacture control. Every device in the relay rack is represented by a symbol on the ladder logic with connections between those devices

- It gets its name from the observation that programs in this particular language resemble ladders with a series of horizontal rungs between two vertical rails.

- This logic consists of contacts that break and make circuits to control coils. Every contact or coil signifies the status of a single bit in the memory of the programmable logic control. A ladder program can refer any number, multiple times to the status of a single bit.

- The "contacts" refer to physical inputs to the PLC from physical devices such as limit switches and pushbuttons.

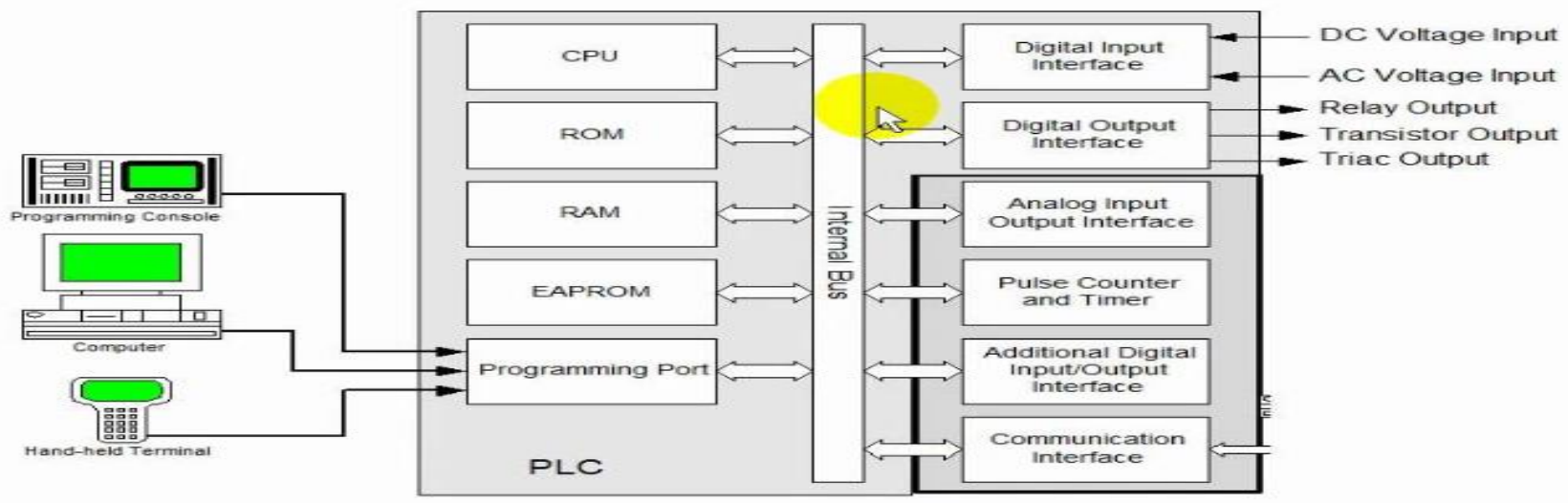

Figure 3.c.1: Block Diagram of PLC

\section{Design Constraints}

We use two software's to simulate and develop the ladder diagram for the pick and place machine. The two software's are genie g-soft and WPL soft by delta. Both software are freeware and are compatible with a wide variety of PLCs. The software's have their own advantages and disadvantages, g- soft provides a better simulation interface but it has a limit of two contacts per rung, which is too few for any real time operation, on the other hand WPL soft doesn't provide a very interactive simulation interface it allows many more contacts to be placed and hence is better suited for larger designs. Design Constraints

The ladder diagram of the pick and place utilizes a number of key elements or design constraints to generate the required logic. We go through certain examples to illustrate these constraints. Lets us consider a basic system in g-soft to go over the first set of constraints.

\begin{tabular}{|c|c|c|c|c|c|c|c|}
\hline & Contact-1 & Connection-1 & Contact-2 & Connection-2 & Contact-3 & Connection-3 & Coil \\
\hline \multirow{2}{*}{01} & \multirow{2}{*}{ start } & & $\mathbf{m l}$ & $\vdots$ & & $\vdots$ & 501 \\
\hline & & & a forward & : & & ; & A. forward \\
\hline \multirow{2}{*}{02} & & & $\mathbf{L} 2$ & $\vdots$ & & $\vdots$ & $-[M 1$ \\
\hline & & & limit switch & $\vdots$ & & $\vdots$ & a forward \\
\hline 03 & & & & & & & \\
\hline & & & & & & & \\
\hline
\end{tabular}

The first example is a simple cylinder system. When the 1st switch is pressed, the cylinder starts moving forward. The operation is stopped when the limit switch I2 is pressed. The system consists of two switches, one output and one auxiliary relay. The auxiliary relay act as an memory element for the plc. Auxiliary relays are similar to the registers present in microprocessors and microcontrollers, but they differ in one key aspect, instead of storing a value, auxiliary relays are used to store a condition or event that occurs. In the example, we can see that the auxiliary relay is used to memorize that the cylinder has reached forward.

When the above ladder diagram is simulated we find that the cylinder stops moving when the switch I 2 is pressed and hence the design is complete. Now let us add a little complexity to the system and say that we define another output that 
we use to retract the cylinder. We also add another limit switch to control the retract action. We extend the design and use M1 to start the operation of retracting the cylinder.

But now we encounter a problem, when the cylinder starts retracting limit switch 1 is released and hence the cylinder will start moving in the forward direction and will be stuck in the forward position. What this indicates is that, the memorizing mechanism deployed needs a hold on circuit. A hold on circuit maintains the state of the circuit even after the input to that circuit is removed. The hold on circuit is nothing but the auxiliary relay M1 that is connected in parallel across I2.

\begin{tabular}{|c|c|c|c|c|c|c|c|}
\hline & Contact-1 & Connection-1 & Contact-2 & Connection-2 & Contact-3 & Connection-3 & Coil \\
\hline \multirow{2}{*}{01} & \multirow[b]{2}{*}{ start } & $\vdots$ & \multirow{2}{*}{${ }_{\text {a forward }} \mathbf{m l}$} & $\vdots$ & & $\vdots$ & \multirow{2}{*}{ A forward } \\
\hline & & & & $\vdots$ & & $\vdots$ & \\
\hline \multirow{2}{*}{02} & & & \multirow{2}{*}{$\begin{array}{l}\mathbf{1 2} \\
\text { limit switch1 }\end{array}$} & $\vdots$ & & $\vdots$ & \multirow{2}{*}{$\begin{array}{l}\text { [ MI } \\
\text { a forward }\end{array}$} \\
\hline & & & & $\vdots$ & & $\vdots$ & \\
\hline \multirow{2}{*}{03} & $\ldots$ & 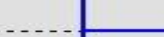 & \multirow{2}{*}{$\begin{array}{l}\text { a forward } \\
\text { M }\end{array}$} & $\vdots$ & \multirow{2}{*}{$-\mathbf{m} 2$} & $\vdots$ & \multirow{2}{*}{ A back } \\
\hline & & & & $\vdots$ & & $\vdots$ & \\
\hline \multirow{2}{*}{04} & & & \multirow{2}{*}{${ }_{\text {limit switch } 2} \mathbf{1 3}$} & $\vdots$ & & $\vdots$ & \multirow{2}{*}{ a back } \\
\hline & & & & $\vdots$ & & $\vdots$ & \\
\hline 05 & & & & & & & \\
\hline
\end{tabular}

\begin{tabular}{|c|c|c|c|c|c|c|c|}
\hline & Contact-1 & Connection-1 & Contact-2 & Connection-2 & Contact-3 & Connection-3 & Coil \\
\hline \multirow{2}{*}{01} & I1 & $\vdots$ & $\mathrm{ml}$ & $\vdots$ & & $\vdots$ & 50 \\
\hline & start & & a forward & $\vdots$ & & $\vdots$ & A forward \\
\hline \multirow{2}{*}{02} & & & -12 & $\vdots$ & & $\vdots$ & $-5 \mathrm{MI}$ \\
\hline & & & limit switch1 & 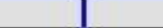 & & $\vdots$ & a forward \\
\hline \multirow{2}{*}{03} & & & MI & & & & \\
\hline & & & a forward & $\vdots$ & & : & \\
\hline \multirow{2}{*}{04} & & & $\mathbf{M I}$ & $\vdots$ & $m 2$ & $\vdots$ & $-\left[\mathrm{O}_{2}\right.$ \\
\hline & & $\vdots$ & a forward & $\vdots$ & a back & $\vdots$ & A back \\
\hline \multirow{2}{*}{05} & MI & $\vdots$ & 13 & $\vdots$ & & $\vdots$ & $-1 \mathrm{M} 2$ \\
\hline & a forward & & limit switch 2 & & & $\vdots$ & a back \\
\hline 06 & . & & $M 2$ & & & & \\
\hline & & $\vdots$ & a back & & & & \\
\hline
\end{tabular}

It is apparent now that the cylinder system functions correctly. We draw the following conclusions from this example-

1. Auxiliary relays are used to memorize certain events and are used to control the flow of the ladder diagram, i.e. they are used to create a sequential execution.

2. We use hold on circuits to complete the memorizing path even after the input condition is removed.

The above example and the conclusions drawn from it are important for the ladder diagram that is developed for the pick and place machine. The sensors used to detect presence of panels or the limit switch attached to suction cups are all basically switches and we need to memorize the instance they are triggered and also memorize that action. The monitoring of conditions needs to occur in a particular order for the desired operation.

Apart from this there is one other major design consideration and that is we need to refresh the memory elements (auxiliary relays) when we are using a nested counter. In the pick and place machine as discussed in previous sections, the input for stack and number of panels is taken from the operator, therefore after each cycle i.e. picking one panel and placing it at the destination, we need to reset all the auxiliary relays to reset the system to ready state.

\section{Programming Considerations:}

- Calibration Steps

- Peripheral Interfacing Considerations

- Memorizing Each Action

- Counting of Planks

- Refreshing of Memory Elements 


\section{Flowchart}
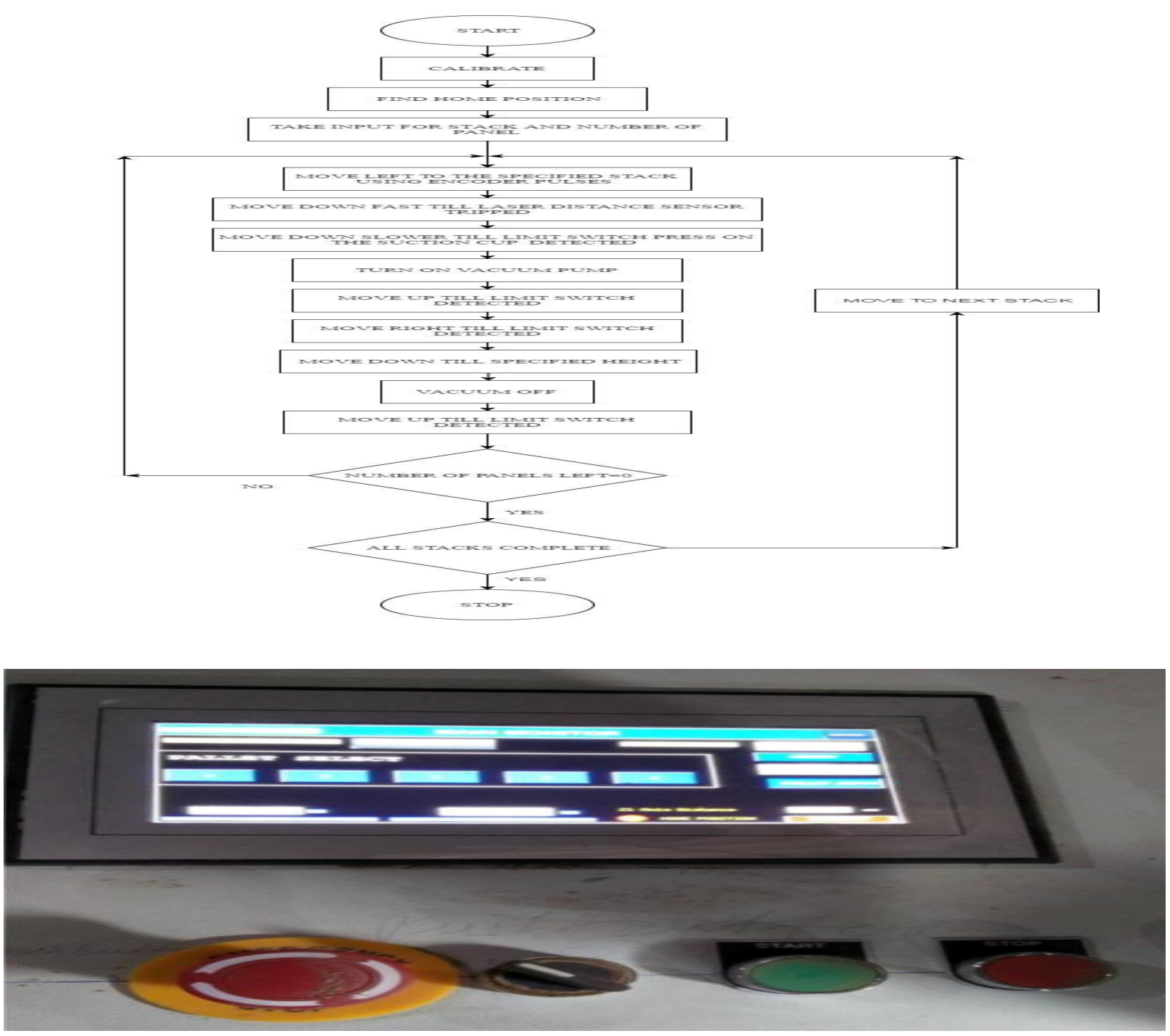

Figure 3.h.2: HMI Board

\subsection{Specifications Of Components Used}

\begin{tabular}{|c|l|l|l|}
\hline$\underline{\mathbf{S . N O}}$. & DESCRIPTION & MAKE & $\underline{\text { RATING }}$ \\
\hline 1. & MCB FORTRANSFORMER & SIEMENS & $25 \mathrm{~A}, 2$ POLE \\
\hline 2. & TRANSFORMER & - & $7.5 \mathrm{KVA}$ \\
\hline 3. & MCB FOR SMPS FAN & SIEMENS & $6 \mathrm{AMP}, 2 \mathrm{POLE}$ \\
\hline 4. & SMPS & SHAVISON & $5 \mathrm{~A}, 24 \mathrm{VDC}$ \\
\hline 5. & MCB FOR SERVO & SIEMENS & $63 \mathrm{~A}, 3 \mathrm{P}$ \\
\hline 6. & SERVO AMPLIFIER & FUJI & $5 \mathrm{KW}$ \\
\hline 7. & SERVO AMPLIFIER & FUJI & $5 \mathrm{KW}$ \\
\hline 8. & SERVO MOTOR & FUJI & $5 \mathrm{KW}$ \\
\hline 9. & SERVO MOTOR & FUJI & $5 \mathrm{KW}$ \\
\hline 10. & TECHNOSHOT HMI7 INCH & FUJI & $24 \mathrm{~V} \mathrm{DC}$ \\
\hline
\end{tabular}




\begin{tabular}{|l|l|l|l|}
\hline 11. & PLC 14DI/10DO & FUJI & 24V DC \\
\hline 12. & PLC CONNECTOR & FUJI & - \\
\hline
\end{tabular}

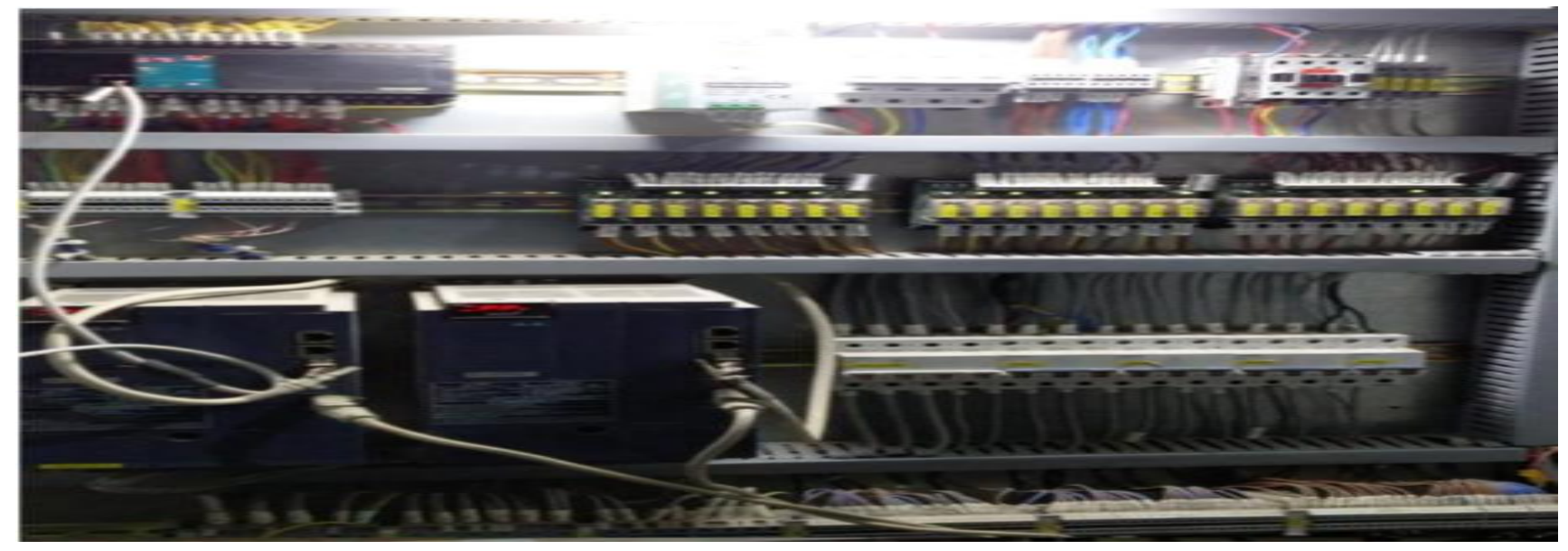

\section{Conclusion}

Figure 3.h.1: Block Diagram of PLC

The wooden planks can be gripped and placed at the required stack within given specified time. The PLC has been programmed correctly and is successfully interfaced and operated for automation of the pick and place machine. The use of human labour to pick up panels has been eliminated, increasing the speed of operation efficiently.

\section{Future Scope}

Automation has caught the world by storm and has always been an ever-growing and flourishing industry. These machines based on PLC have immense scope even from the future perspective, since this has a led to a boom in the automation industry with thunderous results. PLC programming will have varied applications due to the ease in its methodology and numerous advantages.

\section{References}

[1] Dipak Aphale, Vikas Kusekar, "PLC Based Pick And Place Robot", International Journal Of Innovative Research In Science, Engineering And Technology, Vol.5, Issue 2, February 2016.

[2] Jingfujin And Shang Li Yuen. [2013] "A Practical Robotic Grasper With Robust Performance For Pick And Place Tasks" IEEE Transactions On Industrial Electronics, Vol. 60,No.9

[3] 3. S. Chiaverini And L. Sciavicco, (2006) "The Parallel Approach To Force/Position Control Of Robotic Manipulators," IEEE Trans. Robot. Automat., Vol. 9, Pp.361-373.

[4] W. Gruver, "Intelligent robotics in manufacturing, service, and rehabilitation: An overview," IEEE Trans. Ind. Electron., vol. 41, no. 1, pp. 4-11, Feb. 1994

[5] Programmable Logic Controllers Programming methods and applications-Prentice Hall Programmable Logic Controllers (PLCs) (C) 2002 Keyence Corporation

[6] Ray Kulwiec, "Reliability of automated storage and retrieval system", Journal of Institution of Engineers 87 (2006) 26-29.

[7] Liam O' Shea, Development of an "Automated storage and retrieval system in a Dynamic knowledge environment", WaterFord Institute of technology, June 2007.

[8] Barry J., Engineering for "Variety in fully automated Multi production manufacturing system", Wateford institute of technology, 2007.

[9] Bozer Y. A. and White J. A., "Design and performance models for end of Aisle Order picking systems, Management science", v36, N7, 1990.

[10] Marco Ceccarelli,"Low-Cost Robots for Research and Teaching Activities",IEEE Robotics \& Automation Magazine Revised paper no. RAM2001-10-01. of synthetic joint velocity”,ISA Transactions 50 (2011) 131140

[11] Zheng Fang, Yanhua FU, Tianyou Chai, "A Low-cost Modular Robot for Research and Education of Control Systems, Mechatronics and Robotics”, 978-1-4244 (2009) 